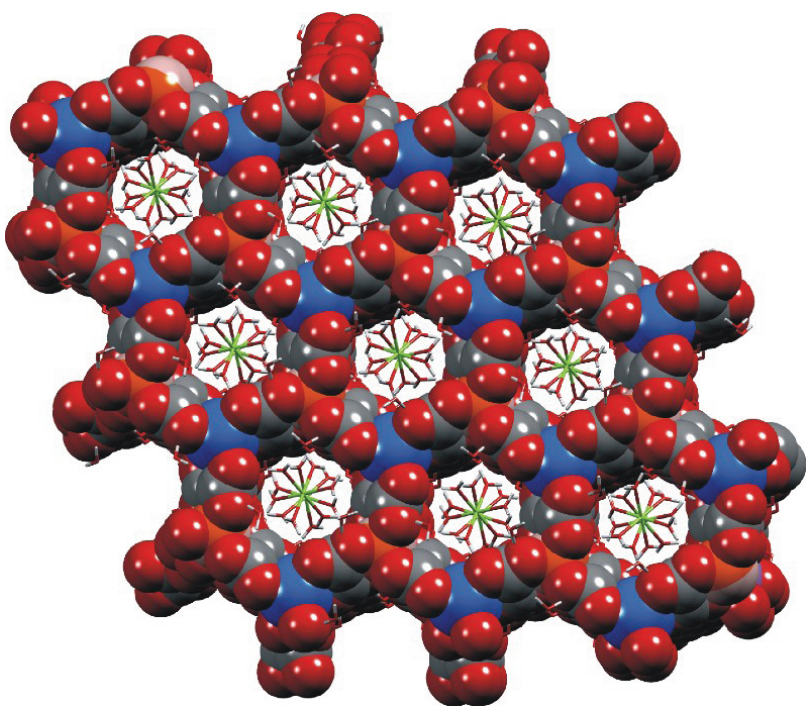

Figure 1. The structure of Zhemchuznikovite viewed down the crystallographic $c$-axis, demonstrating the overlap of neighboring sheets, which leads to the formation of channels occupied by $\mathrm{Mg}\left(\mathrm{H}_{2} \mathrm{O}\right)_{6}{ }^{2+}$ ions.

Keywords: Metal-organic frameworks, minerals, mineral weathering

\section{MS14-O5 A comprehensive analysis of the structure of imogolite nanotubes}

\author{
Stéphan ROUZIERE ${ }^{1}$, Mohamed-Salah AMARA ${ }^{1}$, Erwan \\ PAINEAU $^{1}$, Pascale LAUNOIS ${ }^{1}$
}

1. Laboratoire de Physique des Solides, UMR8502 Université Paris-Sud, Bât. 510, 91405 Orsay Cedex

email: stephan.rouziere@u-psud.fr

Imogolites, these 'tubular clays' with a diameter of the order of the nanometer, discovered in 1962 in volcanic soils [1], can now be synthesized through controllable pathways. They thus appear as most promising candidates for molecular recognition applications taking advantage of their one-dimensional porosity (catalysis, molecular sieves, sensors, etc) [2]. In 1972, natural imogolite nanotubes (INT) were proposed to have nominal composition $(\mathrm{OH})_{3} \mathrm{Al}_{2} \mathrm{O}_{3} \mathrm{SiOH}$, being formed of a curved gibbsite layer whose vacancies are bonded by isolated [Si(Ge)O $\left.\mathrm{O}_{4}\right]$ tetrahedra [3] (figure 1(a)). It was shown later that germanium can replace silicon [4]. Different studies corroborated the structural proposal of Cradwick and co-workers [3] but, to the best of our knowledge, more than forty years after their seminal article, no complete determination of INT structure has been achieved based on experimental data. We present here the first comprehensive X-ray scattering study, from small angles to wide angles, of three sorts of INT, in solution: natural and synthetic $(\mathrm{OH})_{3} \mathrm{Al}_{2} \mathrm{O}_{3} \mathrm{SiOH}$ nanotubes, with different diameters despite their similar composition, and synthetic $(\mathrm{OH})_{3} \mathrm{Al}_{2} \mathrm{O}_{3} \mathrm{GeOH}$ nanotubes [5]. We will explain the strategy we have developed to minimize interatomic distortions and to account for both small and wide angle $\mathrm{X}$-ray scattering data (figure (b)). The refined atomic structures will be discussed with respect to energetical minimizations proposed recently [6]. It is of special interest since these numerical approaches are used as guides to predict new imogolite-like structures [7]. Our methodology should also be applied in the near future to newly-discovered imogolite-type nanotubes (double-walled nanotubes or functionalized nanotubes $[2,8])$.

[1] N. Yoshinaga, S. Aomine, Soil Sci. Plant Nutr. 8, 22 (1962), [2] D.Y. Kang et al, Nat. Comm. 5, 3342 (2014); M.S. Amara et al., Chem. Mater., DOI: 10.1021/cm503428q, [3] P. Cradwick et al, Nature 240, 187 (1972), [4] S. Wada, K. Wada, Clays and Clay Minerals 30, 123 (1982), [5] M.S. Amara, S. Rouzière, E. Paineau, P. Launois, article in preparation, [6] K. Tamura, K. Kawamura, J. Phys. Chem. B 106, 271 (2002); S. Konduri et al, Phys. Rev. B 74, 033401 (2006); L. Guimarães et al, ACS Nano 1, 362 (2007); M. Zhao et al, J. Phys. Chem. C 113, 14834 (2009) ; M.P. Lourenço et al, J. Phys. Chem. C 118, 5945 (2014), [7] L. Guimarães et al, Phys. Chem. Chem. Phys. 15, 4303 (2013), [8] M.S. Amara et al, Chem. Comm. 49, 11284 (2013) 
(a)

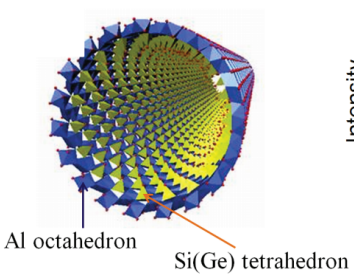

(b)

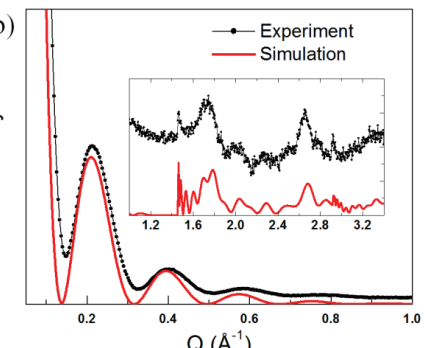

Figure 1. (a) Schematic representation of an imogolite nanotube; (b) X-ray scattering pattern of $(\mathrm{OH})_{3} \mathrm{Al}_{2} \mathrm{O}_{3} \mathrm{GeOH}$ nanotubes, together with its simulation for the refined structure, showing the good agreement between experiment and simulation.

Keywords: Crystallography, imogolite

\section{MS15. Structure property}

relationships

Chairs: Kari Rissanen, Martin Bremholm

MS15-01 Design and synthesis of molecular materials: mixed crystals for finer engineering

Matteo Lusi ${ }^{1}$, Michael J. Zaworotko ${ }^{1}$

1. Chemical and Environmental Science, University of Limerick, Limerick, Ireland

email: matteo.lusi@ul.ie

The properties of a material depend on both the nature of its constituents (chemistry) and the way they are arranged in the space (structure). Crystal engineering is the discipline that investigates and exploits the relationship between structure and physical properties to produce tailor-made crystalline molecular materials. A limitation to such activity is represented by the finite (discrete) nature of the molecular components (building blocks) that prevent the material's fine tuning. By combining the functions and sterics of multiple, equivalent building blocks crystalline solid solutions (mixed crystals), whose composition can be varied in continuum, provide an extra degree of control over the material's properties. Hence, for this class of compounds the crystal engineering problem would coincide with the synthesis of the appropriate mixed crystal. Unfortunately, traditional crystallisation techniques are likely to result in molecule separation precluding the formation of mixed crystal. Therefore solvent-free alternatives must be developed and pursued. Solid-solid and solid-gas reactions represent a viable alternative to wet chemistry in the production of homogeneous solid solutions of organic, metalorganic and organometallic species. This strategy has proved successful in engineering reactivity, polymorphism, photoemission spectra and unit cell metrics of a number of materials including coordination polymers, molecular sieves, dyes and pharmaceuticals.

Keywords: crystal_engineering, solid_solutions, mixed_crystals, solid_state_reactions 